\title{
Identity-by-descent approaches identify regions of importance for genetic susceptibility to hereditary esophageal squamous cell carcinoma
}

\author{
JOSEPHINE MY KO ${ }^{1 *}$, PENG ZHANG ${ }^{2 *}$, SIMON LAW $^{3,5}$, YANHUI FAN $^{4}$, YOU-QIANG SONG ${ }^{4}$, XUE KE ZHAO $^{2}$, \\ ELIBE H.W. WONG ${ }^{1}$, SA TANG $^{2}$, XIN SONG $^{2}$, MARIA LI LUNG ${ }^{1,5}$ and LI DONG WANG ${ }^{2}$ \\ ${ }^{1}$ Department of Clinical Oncology, University of Hong Kong, Hong Kong, SAR; ${ }^{2}$ Henan Key Laboratory for Esophageal \\ Cancer Research of the First Affiliated Hospital, Zhengzhou University, Zhengzhou, Henan; Departments of ${ }^{3}$ Surgery, \\ and ${ }^{4}$ Biochemistry, and ${ }^{5}$ Center for Cancer Research, University of Hong Kong, Hong Kong, SAR, P.R. China
}

Received March 11, 2014; Accepted April 30, 2014

DOI: $10.3892 /$ or.2014.3222

\begin{abstract}
Worldwide, the highest prevalence of esophageal cancer (EC) occurs in Northern China. High-density SNP arrays allow identification of identity-by-descent (IBD) segments in genomic DNAs representative of shared common ancestral regions. We utilized IBD approaches to map susceptibility loci associated with low-penetrance SNPs in high-risk Henan hereditary esophageal squamous cell carcinoma (ESCC) patients. Affymetrix GeneChip Human mapping
\end{abstract}

Correspondence to: Professor Maria Li Lung, Department of Clinical Oncology, Room L6-43, 6/F, Laboratory Block, University of Hong Kong, 21 Sassoon Road, Pokfulam, Hong Kong, SAR, P.R. China

E-mail: mlilung@hku.hk

Professor Li Dong Wang, Henan Key Laboratory for Esophageal Cancer Research of the First Affiliated Hospital, Zhengzhou University, Zhengzhou, Henan, P.R. China

E-mail: ldwang2007@126.com

\section{*Contributed equally}

Abbreviations: ARCL, autosomal recessive cutis laxa; CHB, Han Chinese in Beijing, China; CRC, colorectal cancer; CYP2C18, cytochrome P450, family 2, subfamily C, polypeptide 18; DIRC1, Homo sapiens disrupted in renal carcinoma 1; EC, esophageal cancer; ESCC, esophageal squamous cell carcinoma; $\mathrm{FH}^{-}$, family history-negative; $\mathrm{FH}^{+}$, family history-positive; $G A P D H$, glyceraldehyde-3-phosphate dehydrogenase; GPT2, glutamic pyruvate transaminase (alanine aminotransferase 2); GULP1, Homo sapiens GULP, engulfment adaptor PTB domain containing 1; GWAS, genome-wide association study; HWE, Hardy-Weinberg equilibrium; IBD, identity-by-descent; LOH, loss of heterozygosity; MAF, minor allele frequency; NT, non-tumor; PLCE1, phospholipase C, epsilon 1; SIAHI, Siah E3 ubiquitin protein ligase 1; T, tumor; UPL, universal probe library

Key words: IBD, inherited ESCC, genetic susceptibility, SNP array, Chinese
SNP array IBD analysis was performed in 32 Henan family history-positive $\left(\mathrm{FH}^{+}\right)$ESCC patients, 18 Henan healthy unrelated individuals, and 45 Chinese individuals from a CHB HapMap dataset using PLink (scoring IBD segments individually) and BEAGLE (scoring of shared IBD segments among case/case vs. control/control pairs) software. Both analyses identified longer IBD segment lengths associated with $\mathrm{FH}^{+}$ESCC compared to controls. However, there was no strong evidence for a genetic founder effect. Pairing IBD analysis with BEAGLE identified 8 critical IBD segments residing at 2q32.1-q32.2, 3p22.3-p22.2, 4q21.1-q21.21, 7p22.2, 8q23.2-q23.3, 10q23.33-q24.1, 14q24.3 and 16q11.2-q12.1, which were more significantly shared among case/case compared to control/control. The shared IBD segments in $\mathrm{FH}^{+}$ ESCC samples with no overlap with control/CHB HapMap may encompass potential cancer susceptibility loci. Selected targeted genes, PLCE1, GPT2, SIAH1 and CYP2C-18, residing within the IBD segments at 10q23.33-q24.1 and 16q11.2-q12.1, had statistically significant differential expression in primary ESCC tissues and are likely involved in ESCC carcinogenesis. The importance of these IBD segments to the etiology and development of ESCC in high-risk areas requires further study with expanded sample sizes. This is the first report employing the pairing IBD approach for elucidation of the genetic basis of hereditary ESCC in Henan by applying high throughput SNP array analysis.

\section{Introduction}

Esophageal cancer (EC) is the third most prevalent gastrointestinal malignancy in the world. It is a deadly disease with 5-year survival rates ranging from 10 to $16 \%$ (1). Esophageal squamous cell carcinoma (ESCC) is the major histologic subtype of EC. Annually, there are 462,000 reported new cases and 386,000 deaths; $75 \%$ of the patients die within one year of diagnosis (1). The incidence of EC shows great geographical variation. Its high prevalence is of particular importance in Northern China. Worldwide, the south side of the Taihang Mountains in North-central China along the northern borders of Hebei, Henan and Shanxi Provinces forms one of the 
highest risk regions of EC (2-4). In Linzhou County of Henan Province in 1993-1997, the age-standardized incidence rate of EC in males and females was 121/100,000 and 80/100,000, respectively (5). Epidemiological studies suggest that environmental factors such as alcohol abuse, tobacco smoke and dietary carcinogens may play a substantial role in the etiology of EC (6,7). However, strong evidence from familial aggregation of EC in Yangcheng County and segregation analysis of EC in Yangquan indicate the existence of genetic susceptibility loci for EC predisposition $(3,8)$. Historically, the Chaoshan people in Nanao, which is another high-risk region of EC in Southern China (9), originally migrated from the Taihang Mountain region. The distinctly high EC incidence in the Chaoshan population further strengthens the etiologic role played by genetic components in familial EC. A third line of evidence supporting a causative role of genetic factors in familial EC comes from molecular genetic studies of loss of heterozygosity (LOH), SNP association, microarray profiling and BRCA2 germline mutations (10-14).

Recent technological advances allow the identification of long regions of homozygosity in genomic DNAs using high-density SNP arrays. These homozygous regions, which may dictate the level of consanguinity of an individual, are referred to as identity-by-descent (IBD) segments, also known as autozygosity $(15,16)$. The IBD segments represent the sharing of common sequences from an ancestor in those regions. Previous studies suggest that a high rate of consanguinity, which produces germline genomic homozygosity, is associated with cancers (17-20). A recent study using SNP array analysis showed that the signatures of autozygosity correlate with colorectal cancer (CRC) incidence, and the IBD regions may help localize the genes that contribute to CRC heritability (15). However, IBD regions are difficult to locate without pedigree information. We hypothesized that the IBD approach is useful for mapping the common susceptibility locus with low-penetrance SNPs in hereditary ESCC patients in Henan, one of the highest ESCC risk regions in the world. Screening programs based on knowledge of the candidate cancer susceptibility locus may reduce cancer mortality by prevention. Thus, the long-term aim is to better understand the genetic basis for ESCC in Henan to achieve the ultimate goal of improving ESCC patient survival.

\section{Materials and methods}

Samples for SNP analysis. Samples for IBD analysis consisted of 50 blood samples from 18 healthy unrelated individuals and $32 \mathrm{FH}^{+}$ESCC patients from the high-risk region of Henan Province and nearby counties from the Linzhou Center Hospital and Yaocun Esophageal Cancer Hospital collected from 2001 to 2008. Table I summarizes the clinical information of the 32 cases and 18 controls and details of the family history of the ESCC patients. Thirty-one of the $32 \mathrm{FH}^{+}$ patients analyzed involved at least two generations having a family history of ESCC. Approval for use of human blood and/ or information was obtained from the Committee for Ethical Review of Research Involving Human Subjects at Zhengzhou University. The study was conducted according to Declaration of Helsinki principles. Informed written consent was obtained from each individual.
DNA extraction from clotted blood. Between 0.4 and $2 \mathrm{ml}$ of clotted blood was used for DNA extractions. DNA extraction was performed as described by Kanai et al (21). Briefly, $250 \mu \mathrm{l}$ of lysis solution was mixed with each $200 \mu \mathrm{l}$ of clotted blood and incubated overnight at $55-65^{\circ} \mathrm{C}$. Phase separation was performed by centrifugation, and DNA was precipitated in ethanol.

SNP array and genotyping procedure. The SNP genotyping was performed at the UCLA microarray center with Affymetrix GeneChip Human mapping ( 248K SNPs) Sty I SNP Array (Affymetrix). Genotypes were called by the GeneChip DNA Analysis Software (v2.0, Affymetrix).

CHB HapMap genotype data. Genotype data of Affymetrix GeneChip Human mapping ( 238K SNPs) Sty I SNP Array of 45 unrelated individuals from Han Chinese in Beijing, China (CHB), which is publicly accessible, were downloaded from the Affymetrix technical documentation (http://www.affymetrix. com/support/technical/sample_data/500k_hapmap_genotype_data.affx).

Quality control. A total of 238,032 SNPs were filtered with a SNPs call rate $\geq 0.90$, individual call rate $\geq 0.94$, and a minor allele frequency $(\mathrm{MAF})>0.05$. SNPs were selected to have Hardy-Weinberg equilibrium (HWE), P-values of $\geq 0.001$ in controls and the CHB HapMap dataset. After applying these stringent quality control measures for the inherited ESCC IBD study, genotypes for 150,940 SNPs were available for $28 \mathrm{FH}^{+}$ cases, 16 controls and a 45 CHB HapMap dataset.

Statistical and bioinformatic analysis. PLink (22) (v1.06) software (http://pngu.mgh.harvard.edu/ purcell/plink/contact. shtml\#cite) was used to detect IBD segments of different combinations with a threshold limit of IBD segments ranging from 1 to $4 \mathrm{Mb}$ in length and comprised of 20-50 SNPs in each individual's genome. The threshold limit of IBD segments was defined as a stretch of runs of at least e.g. 50 consecutive homozygous SNPs encompassing a minimum of e.g. $2 \mathrm{Mb}$. Remaining options were set to default values. BEAGLE software was used to detect the sharing of IBD regions in paired individuals (23). A fast IBD score $<10^{-10}$ and the shared haplotype length of $\geq 1 \mathrm{Mb}$ were used as thresholds to indicate strong evidence of the shared IBD haplotypes among the genomes of pairs of individuals (case/case vs. control/ control pairing). Comparisons of the difference in the average lengths of IBDs between cases, controls and CHB HapMap dataset were performed by ANOVA. Statistical analysis for comparison of the distribution of categorical variables was performed by Chi-square test and Fisher's exact test in the pair-IBD approach.

ESCC tissue specimens and real-time quantitative PCR analysis. Total RNAs from 31 pairs of matched esophageal epithelial non-tumor and tumor specimens from ESCC patients were extracted by TRIzol reagent as previously described (24). These included 11 Henan $\mathrm{FH}^{+}, 10$ Henan family $\mathrm{FH}^{-}$, and 10 Hong Kong ESCC specimens. Henan and Hong Kong ESCC specimens were collected from Yaocun Esophageal Cancer Hospital, Linzhou, Henan, 
Table I. Clinical information and details of the family history of the ESCC patients.

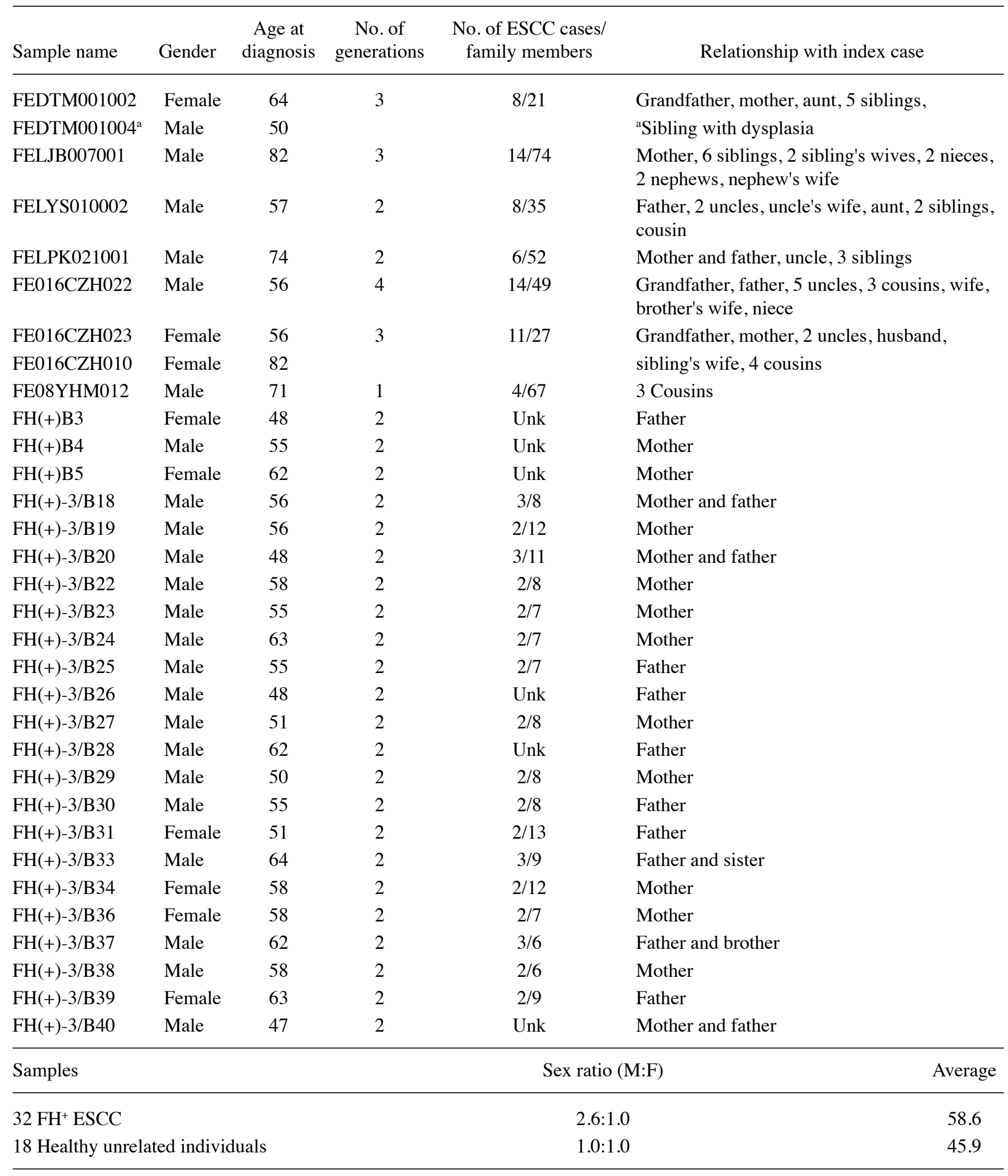

China from 2005 to 2007 and Queen Mary Hospital from 2004 to 2008, respectively. Approval for the present study was obtained from the Hospital Institutional Review Board at the University of Hong Kong, Hong Kong. Two micrograms of total RNA was reverse transcribed into cDNAs by SuperScript III reverse transcriptase (Invitrogen), as described in the manufacturer's manual. Real-time qPCR was performed in a StepOnePlus machine (Applied Biosystems) using FastStart Universal Probe Master Mix (Roche Diagnostics) and glyceraldehyde-3-phosphate dehydrogenase (GAPDH) TaqMan probes (Applied Biosystems). The universal probe library (UPL) (Roche Diagnostics) was 
A

Case

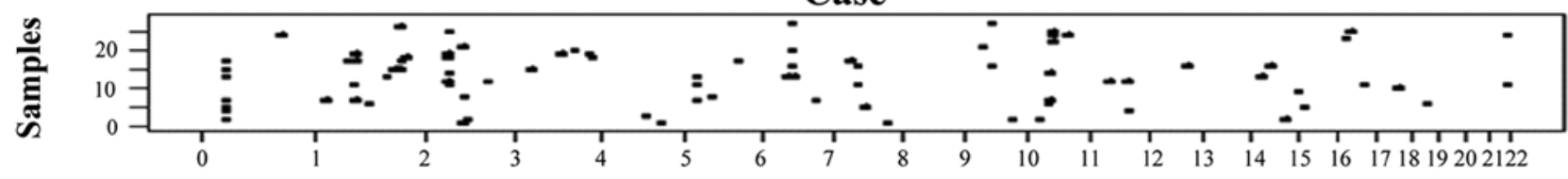

Control

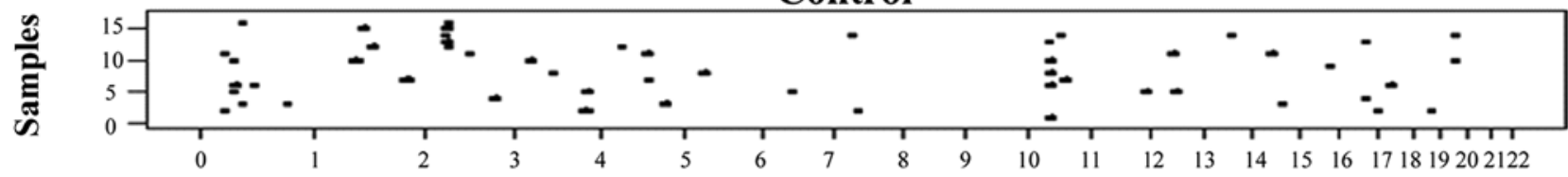

Hapmap

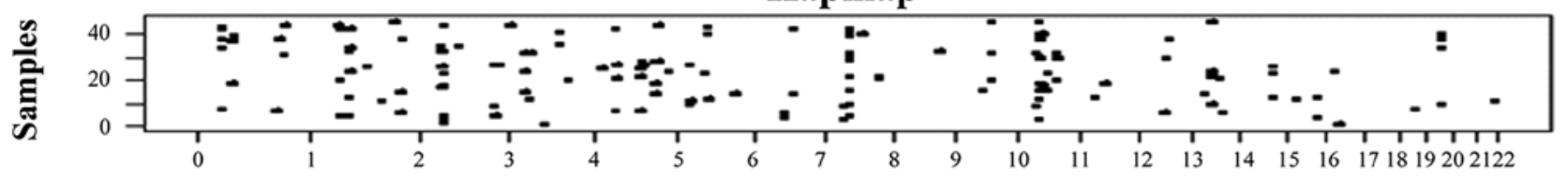

Chromosome

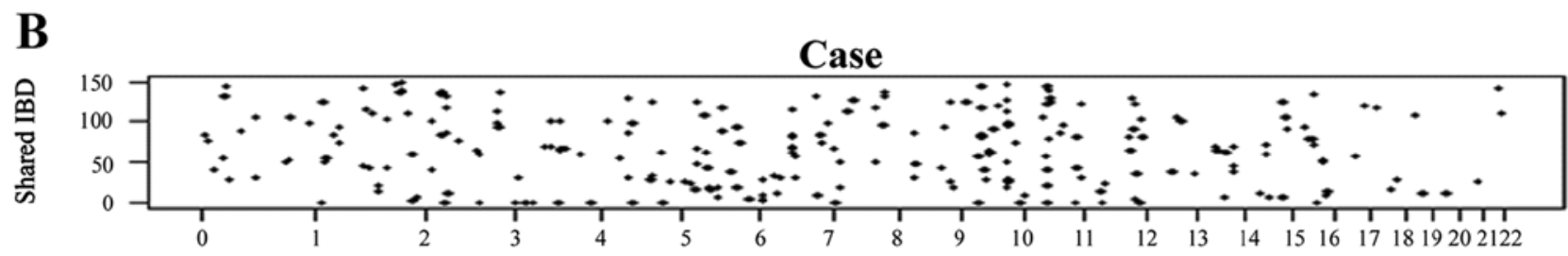

Control

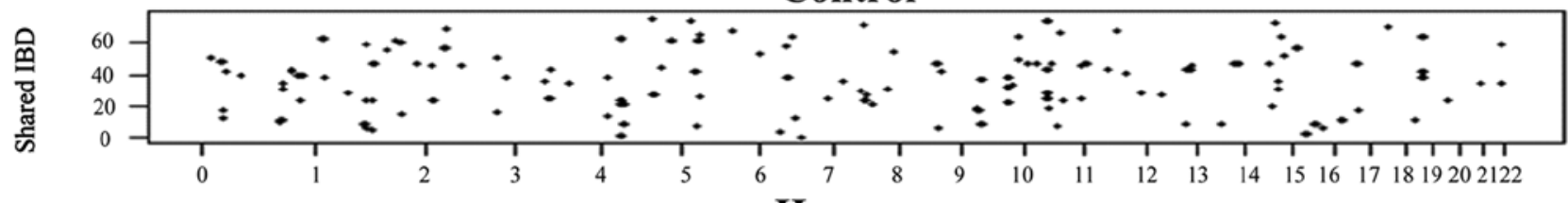

Hapmap

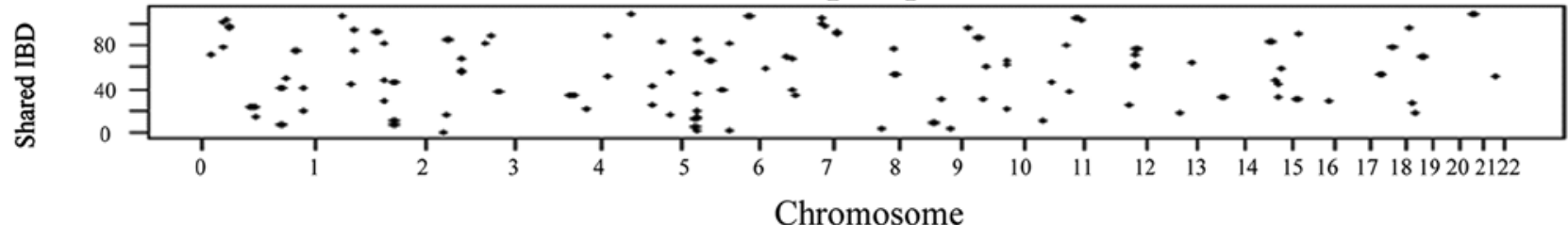

Figure 1. IBD segment lengths. Genome-wide plots of (A) IBD segment distribution identified by the PLink analysis. The threshold was set to a minimum of $2 \mathrm{Mb}$ in length containing at least 50 consecutive homozygous SNPs. (B) Shared IBD segment distribution identified by the pairing analysis with BEAGLE, among the genomes of $28 \mathrm{FH}^{+}$Henan ESCC patients (case), 16 control individuals from Henan, and 45 individuals from the CHB HapMap datasets. The BEAGLE pairing approach was superior in identification of the IBD segments among the Henan ESCC cases compared to the controls or CHB HapMap.

used to validate the expression of PLCE1 (NM_016341.3), CYP2-C18(NM_000772.2),SIAH1 (NM_001006610.1) and GPT2 (NM_001142466.1), as previously described (25). Primer sequences and UPL probe number used are provided upon request.

\section{Results}

IBD segments: individual germline genomic homozygosity with exceptionally long stretches of SNPs. The germline homozygosity in 28 Henan $\mathrm{FH}^{+}$ESCC patients was explored by analysis with Affymetrix GeneChip Human mapping SNP array ( 238K SNPs, Sty I). The IBD segment approach scores the individual germline homozygosity (IBD segments) by detection of long stretches of at least $2 \mathrm{Mb}$ containing a minimum of 50 consecutive homozygous SNPs in the blood DNAs. These IBD segments were distributed across the genomes of the $28 \mathrm{FH}^{+}$ESCC cases, 16 Henan normal controls and 45 individuals from the CHB HapMap datasets (Fig. 1A). The threshold was set to a minimum of $2 \mathrm{Mb}$ in length containing at least 50 consecutive homozygous SNPs. Longer average IBD segment length and higher average number of SNPs in IBD segments were also detected in the cases compared to the controls and CHB HapMap controls, when the threshold was set ranging from 20 to 50 consecutive homozygous SNPs (data not shown). A total of 26 IBD segments in the $\mathrm{FH}^{+}$ESCC samples having no overlap with the control and CHB HapMap were detected (data not shown). The physical map is based on the Human Feb. 2009 (GRCh37/ hg19) assembly (http://genome.ucsc.edu/cgi-bin/hgGateway). 

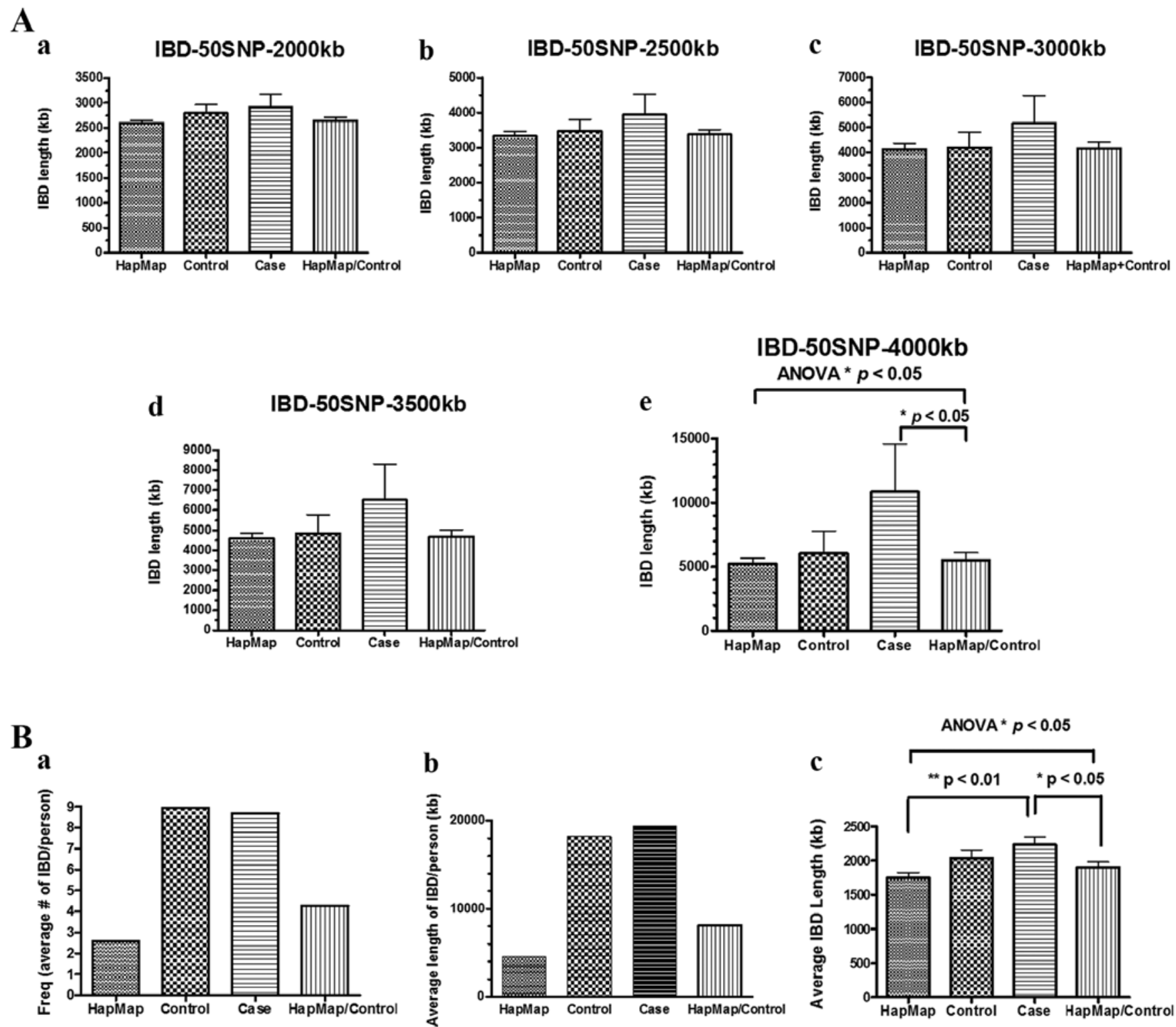

Figure 2. Longer average IBD segments in Henan $\mathrm{FH}^{+}$ESCC patients. Comparisons of IBD lengths by scoring IBD segments individually (A) and pairing IBD analysis. (A) (a-e) Comparisons of IBD lengths in the $\mathrm{FH}^{+}$ESCC patients $(\mathrm{n}=28)$ showed a trend of longer average IBD segments compared to the Henan controls ( $n=16)$, CHB HapMap ( $n=45)$ and Henan control/CHB HapMap $(n=61)$, when the threshold was set ranging from 2 to 4 Mb in length containing at least 50 consecutive homozygous SNPs. (B) (a) Frequency (number of IBD segments per person) using the homozygosity mapping approach for bioinformatic analysis of case/case and control/control pairing, and (b) the average length of IBD per person was higher in the cases compared to the CHB HapMap dataset, but similar in the cases and the controls. (c) The $\mathrm{FH}^{+}$ESCC patients had statistically significant longer average IBD lengths compared to CHB HapMap alone and CHB HapMap/control. A longer length of IBD segments was also observed, when a comparison was carried out between the case and control, although the difference did not achieve statistical significance.

Among the $28 \mathrm{FH}^{+}$ESCC samples, patient FH3B31 had the longest total length of $29.3 \mathrm{Mb}$ IBD segments distributed in 6 regions. Patient FH3B24 had the longest IBD segment of $21.1 \mathrm{Mb}$ encompassing $799 \mathrm{SNPs}$ at chromosome 2q31.1q32.2. When the IBD segment was defined as a minimum of $2 \mathrm{Mb}$ in length containing at least 50 consecutive homozygous SNPs, 22/28, 14/16 and 38/45 (78.6, 87.5 and 84.4\%) of $\mathrm{FH}^{+}$ ESCC patients, Henan controls and CHB HapMap controls were observed as having more than one IBD segment, respectively. However, a trend of longer average IBD segments, but not a higher frequency of IBD segments, was observed in $\mathrm{FH}^{+}$ ESCC patients compared to the Henan controls and Henan
control/CHB HapMap, when the threshold was set ranging from 2 to $3.5 \mathrm{Mb}$ in length containing at least 50 consecutive homozygous SNPs, although the difference did not reach statistical significance (Fig. 2A-a-d). $\mathrm{FH}^{+}$ESCC patients had statistically significant longer average IBD segments compared to the Henan control/CHB HapMap, when the threshold was set to $4 \mathrm{Mb}$ in length containing at least 50 consecutive homozygous SNPs (Fig. 2A-e).

Pairing analysis for sharing of IBD segment approach: eight critical regions with significantly higher frequencies of sharing of IBD segments among the $\mathrm{FH}^{+}$ESCC patients 


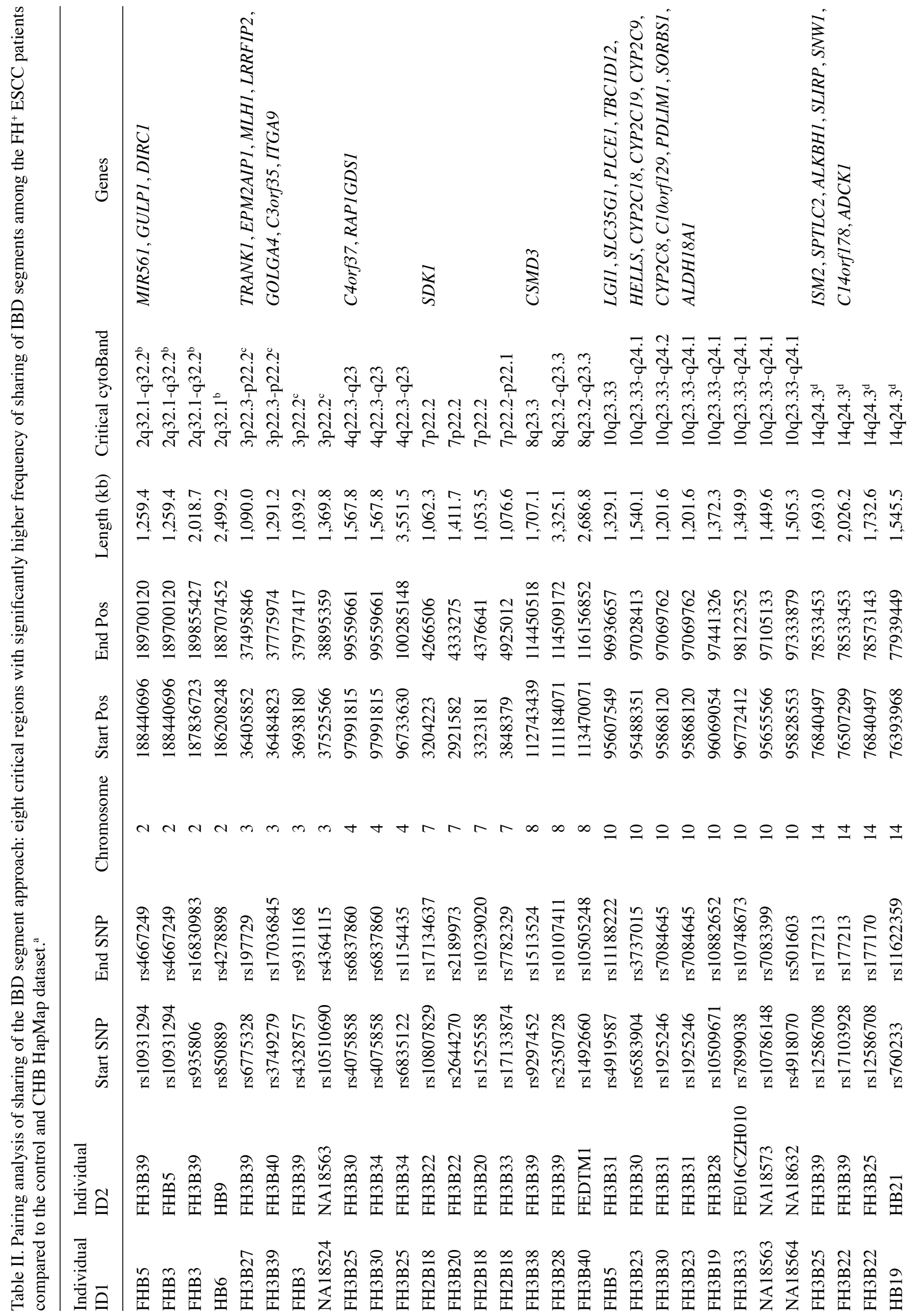


compared to the CHB HapMap/Henan normal datasets. The pairing analysis approach, performed with the BEAGLE software, was used for identification of the shared IBD segments distributed across the genomes of the $\mathrm{FH}^{+} \mathrm{ESCC}$ cases, Henan normal controls, and individuals from the CHB HapMap datasets (Fig. 1B). When the bioinformatic analysis was carried out based on the homozygosity mapping approach of case/case and control/control pairing, the frequency (number of IBD segments per person) and average length of IBD per person were higher in the cases compared to the CHB HapMap dataset, but similar in the cases and controls from Henan (Fig. 2B-a and -b). The $\mathrm{FH}^{+}$ESCC patients had statistically significant longer average IBD lengths compared to the CHB HapMap alone and the CHB HapMap/Control. A longer length of IBD segments was also observed, when a comparison was made between the cases and controls, although the difference did not reach statistical significance (Fig. 2B-c).

Among the 28, 16 and 45 individuals in the case, control, and CHB HapMap groups, the numbers of pairs for calculation of sharing events were 378,120 and 990, respectively. Table II summarizes the 8 critical regions that are shared at a statistically significant higher number of events in the $\mathrm{FH}^{+}$ESCC cases compared to the CHB HapMap controls. The 8 critical regions include 2q32.1-q32.2, 3p22.3-p22.2, 4q21.1-q21.21, 7p22.2, 8q23.2-q23.3, 10q23.33-q24.1, 14q24.3 and 16q11.2-q12.1. The genes involved are also listed in Table II. Table III summarizes the statistical analysis of the eight critical IBD regions in Henan $\mathrm{FH}^{+}$ESCC identified by excessive sharing of homozygous segments in the case/case and control/control pairing. These regions include $7 \mathrm{p} 22.2$ with four and zero sharing events of IBD segments; six regions at 2q32.1-q32.2, 3p22.3-p22.2, 4q21.1q21.21, 8q23.2-q23.3, 14q24.3 and 16q11.2-q12.1 were detected to have three and zero sharing of IBD segments and a critical region at 10q23.33-q24.1 was detected to have six and two sharing events of IBD segments out of 378 and 990 pairing events in the cases and CHB HapMap dataset, respectively (Table III).

Validation of target gene expression identified by pairing IBD analysis by quantitative RT-PCR. To examine the differential gene expression profiling among familial ESCC cases from high-risk Mainland China and cases from moderate-risk Hong Kong, an oligonucleotide microarray analysis with the $28 \mathrm{~K}$ gene chip prepared at the Genome Institute of Singapore, as previously described (26), was performed using 31 pairs of ESCC specimens from Henan $\left(11 \mathrm{FH}^{+}, 10 \mathrm{FH}^{-}\right)$and Hong Kong (10 cases) (data not shown). Four critical target genes at 10q23.33-q24.1 and 16q11.2-q12.1 (Table II) were chosen for further study to demonstrate their clinical and biological relevance after comparison for overlap between the two lists of candidate genes identified by pairing IBD analysis and microarray differential expression. Validation of target gene expression by quantitative RT-PCR demonstrated significant overexpression of PLCE1 and SIAH1, as well as downregulation of $C Y P 2-C 18$ and GPT2 expression using GAPDH expression for normalization (Fig. 3). Among the 31 pairs of ESCC cases, the frequencies of PLCEI and SIAHI overexpression were $64.5(20 / 31)$ and $90.0 \%$ (27/30), respectively, while the frequencies of GPT2 and CYP2-C18 downregulation were $80.6(25 / 31)$ and $76.7 \%(23 / 30)$ in ESCC primary tumor tissues, respectively, when the threshold was set at a 2 -fold difference. 
Table III. Statistical analysis of the eight critical IBD regions in Henan $\mathrm{FH}^{+}$ESCC cases identified by excessive sharing of homozygous segments in case/case and control/control pairing.

\begin{tabular}{llccccc}
\hline & Critical IBD & $\begin{array}{c}\text { Frequency of IBD } \\
\text { sharing events } \\
\text { in cases, } \\
\text { region }\end{array}$ & $\begin{array}{c}\text { Frequency of IBD } \\
\text { sharing events } \\
\text { in controls } \\
(\mathrm{n}=16)\end{array}$ & $\begin{array}{c}\text { P-value } \\
\text { (Fisher exact test, } \\
\text { 2-tailed) }\end{array}$ & $\begin{array}{c}\text { Frequency of IBD } \\
\text { sharing events in } \\
\text { CHB HapMap } \\
\text { (n=45) }\end{array}$ & $\begin{array}{c}\text { P-value } \\
\text { (Fisher exact test, } \\
\text { 2-tailed) }\end{array}$ \\
\hline 1 & 2q32.1-q32.2 & $3 / 378$ & $0 / 120$ & 1.0000 & $0 / 990$ & $\mathbf{0 . 0 2 1 0}$ \\
2 & 3p22.3-p22.2 & $3 / 378$ & $0 / 120$ & 1.0000 & $0 / 990$ & $\mathbf{0 . 0 2 1 0}$ \\
3 & 4q21.1-q21.21 & $3 / 378$ & $0 / 120$ & 1.0000 & $0 / 990$ & $\mathbf{0 . 0 2 1 0}$ \\
4 & 7p22.2 & $4 / 378$ & $0 / 120$ & 0.5768 & $0 / 990$ & $\mathbf{0 . 0 0 5 8}$ \\
5 & 8q23.2-q23.3 & $3 / 378$ & $0 / 120$ & 1.0000 & $0 / 990$ & $\mathbf{0 . 0 2 1 0}$ \\
6 & 10q23.33-q24.1 & $6 / 378$ & $0 / 120$ & 0.3436 & $2 / 990$ & $\mathbf{0 . 0 0 7 1}$ \\
7 & 14q24.3 & $3 / 378$ & $0 / 120$ & 1.0000 & $0 / 990$ & $\mathbf{0 . 0 2 1 0}$ \\
8 & 16q11.2-q12.1 & $3 / 378$ & $0 / 120$ & 1.0000 & $0 / 990$ & $\mathbf{0 . 0 2 1 0}$ \\
\hline
\end{tabular}
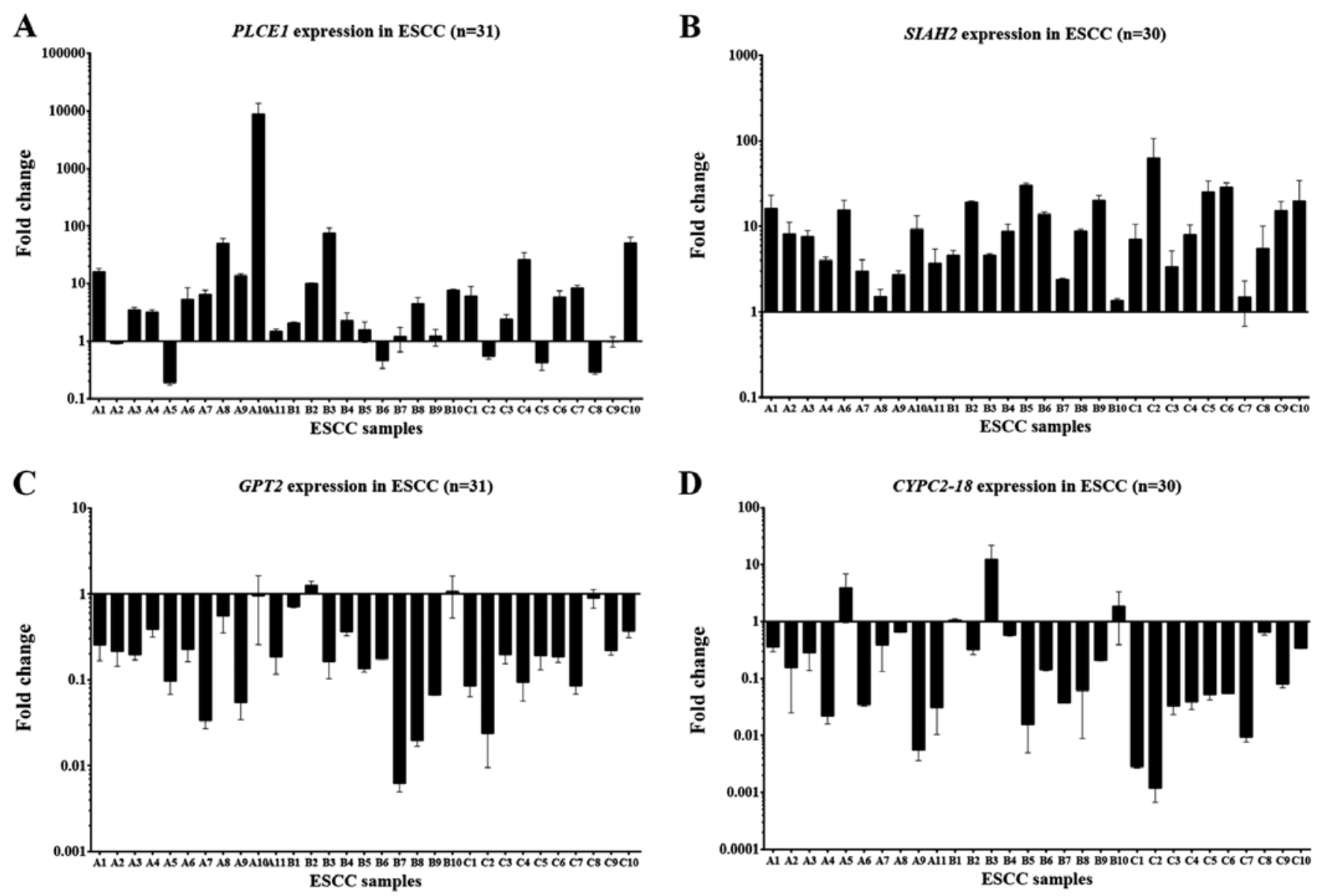

Figure 3. Expression levels of four candidate genes. Real-time qPCR demonstrated significantly overexpressed (A) PLCE1 (20/31, 64.5\%), (B) SIAH1 (27/30, $90.0 \%)$ and downregulated (C) GPT2 (25/31,80.6\%), (D) CYP2-C18 (23/30, 76.7\%) expression in ESCC primary tumor tissues using a threshold of 2-fold difference.

\section{Discussion}

Increased risk of cancer incidence has been observed in inbred populations $(18,20,27)$. An inbred population is characterized by the sharing of longer IBD segments. These observations raise the question as to whether germline homozygosity is involved in cancer predisposition, particularly in inherited cancer. Technological advances in SNP array provide the opportunity to address this issue by the identification of these long stretches of IBD segments among cancer patients. Homozygosity mapping was reported as an efficient strategy to map human recessive traits with the DNA of affected children from consanguineous marriages $(28,29)$. The information obtained from a single affected child of a first cousin 
Table IV. Summary of the results of the IBD regions identified by individual homozygosity mapping and sharing of homozygous segments in case/case and control/control pairing approaches.

Approaches

\begin{tabular}{|c|c|c|c|c|c|c|c|c|}
\hline \multirow[b]{3}{*}{ Data set } & \\
\hline & \multicolumn{4}{|c|}{ Homozygosity mapping (50 SNPs, $2 \mathrm{Mb}$ ) } & \multicolumn{4}{|c|}{ Pairing of IBD segments } \\
\hline & $\begin{array}{l}\text { Total IBD } \\
\text { length } \\
(\mathrm{Mb}) / \mathrm{no} . \\
\text { of IBD } \\
\text { segments }\end{array}$ & $\begin{array}{l}\text { Average } \\
\text { IBD } \\
\text { length } \\
(\mathrm{Mb})^{\mathrm{a}}\end{array}$ & $\begin{array}{c}\text { Average } \\
\text { IBD } \\
\text { length } \\
(\mathrm{Mb}) / \text { person }\end{array}$ & $\begin{array}{l}\text { Frequency } \\
\text { of IBD } \\
\text { segments }\end{array}$ & $\begin{array}{c}\text { Total IBD } \\
\text { length } \\
(\mathrm{Mb}) / \mathrm{no} . \\
\text { of IBD } \\
\text { segments }\end{array}$ & $\begin{array}{l}\text { Average } \\
\text { IBD } \\
\text { length } \\
(\mathrm{Mb})^{\mathrm{a}}\end{array}$ & $\begin{array}{c}\text { Average } \\
\text { IBD } \\
\text { length } \\
(\mathrm{Mb}) / \text { person }^{\mathrm{b}}\end{array}$ & $\begin{array}{l}\text { Frequency } \\
\text { of IBD } \\
\text { segments }\end{array}$ \\
\hline CHB HapMap, n=45 & $378.7 / 146$ & 2.59 & 8.42 & 3.2 & 204.3/117 & 1.75 & 4.54 & 2.6 \\
\hline Henan control, $n=16$ & $156.5 / 56$ & 2.79 & 9.78 & 3.5 & $291.1 / 143$ & 2.04 & 18.20 & 8.9 \\
\hline $\mathrm{FH}^{+} \mathrm{ESCC}, \mathrm{n}=28$ & $239.4 / 82$ & 2.92 & 8.55 & 2.9 & $542.4 / 243$ & 2.23 & 19.37 & 8.7 \\
\hline
\end{tabular}

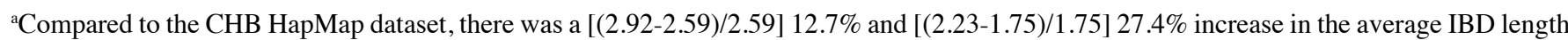
$(\mathrm{Mb})$ in $\mathrm{FH}^{+}$ESCC using homozygosity mapping (PLink) and pairing of IBD segments (BEAGLE) approaches, respectively. ${ }^{\mathrm{b}} \mathrm{Compared}$ to the CHB HapMap dataset, there was an $\sim 4.3$-fold increase in the average IBD length (Mb)/person in the Henan control and $\mathrm{FH}^{+} \mathrm{ESCC}^{\mathrm{c}} \mathrm{Compared}$ to the CHB HapMap dataset, there was an $\sim 3.3$-fold increase in the frequency of IBD segments in the Henan control and FH ${ }^{+}$ESCC.

marriage is the same as those using linkage mapping with a nuclear family with three affected children. By studying DNA of less than a dozen unrelated, affected inbred individuals, homozygosity mapping makes it possible to map recessive diseases (28). An example of the application of homozygosity mapping of five unrelated consanguineous families with autosomal recessive cutis laxa (ARCL) identified a candidate region on chromosome $17 \mathrm{q} 25$, and disease-causing mutations in PYCRl were detected by high-throughput sequencing of the candidate region (29).

China is one of the highest risk regions of EC worldwide; the reported age-standardized incidence rate of EC in males and females was 37.9/100,000 and 12.0/100,000, respectively, in 2002 (1). The Taihang-Hebei-Henan-Shanxi area in Northcentral China forms an extraordinarily high risk region for this type of cancer (2-4). The age-standardized incidence rate of EC in males and females was 23 -fold and 6.5-fold higher, respectively, in Linzhou County of Henan Province in 19931997 compared to that in other parts of China (5). There was an $\sim 10$-fold increase in esophageal carcinoma incidence in these highest risk regions of the Taihang-Hebei-Henan-Shanxi area compared to a moderate-risk region such as Hong Kong. The present study used the homozygosity mapping approach of affected ESCC patients from those villages with exceptionally high incidence of ESCC. Consanguineous marriages in these villages were highly suspected as longer average IBD segments were observed in Henan villages among both the controls and cases (Fig. 2B-a and -b; Table IV). The presence of homozygous segments in an individual's genome can be explained by tracing one's parental lineage to a common ancestor. The first approach identified 26 non-overlapping IBD segments that fulfilled a threshold criteria set (50 SNPs, $2 \mathrm{Mb}$ ) in the present study of $\mathrm{FH}^{+}$ESCC cases from Henan. Two individuals are identical by descent at a locus, when they share the same genetic materials from a common ancestor. The second approach in the present study detected excessive sharing of eight critical IBD regions in the genomes between two affected individuals with $\mathrm{FH}^{+}$ESCC. Table IV summarizes the results of IBD regions identified by individual homozygosity mapping and shared IBD segments in the case/case and control/control pairing approaches. Both approaches detected longer average IBD lengths in $\mathrm{FH}^{+}$ESCC cases, when compared to the $\mathrm{CHB}$ HapMap dataset (Table IV). In the second approach, compared to the CHB HapMap dataset, there was a $\sim 4$.3-fold increase in the average IBD length per person in the Henan controls and $\mathrm{FH}^{+} \mathrm{ESCC}$ cases. There was an $~ 3.3$-fold increase in the frequency of IBD segments in the Henan controls and $\mathrm{FH}^{+}$ ESCC cases compared to the CHB HapMap dataset. The data suggested that populations from Henan villages (both $\mathrm{FH}^{+}$ ESCC and controls) showed a higher rate of consanguinity compared to the CHB HapMap dataset. The critical regions detected by the pair-wise comparison provided additional information on the potential cancer susceptibility loci for ESCC development and should be further studied with a few affected inbred individuals or a higher number of $\mathrm{FH}^{+} \mathrm{ESCC}$ patients.

The only overlapping region, which may predispose an individual to develop ESCC, identified by both approaches was at 2q31.1-q32.2, in which a region of $21.1 \mathrm{Mb}$ (chr 2: 170,290,090-191,418,198) identified by IBD detected in each individual alone was further narrowed down to $1.26 \mathrm{Mb}$ containing one microRNA, MIR561, and two candidate genes Homo sapiens GULP, engulfment adaptor PTB domain containing 1 (GULPl), and Homo sapiens disrupted in renal carcinoma 1 (DIRC1) (chr 2: 188,440,696-189,700,120) by considering pairing information in the latter approach. GULP1 is an adapter protein necessary for the engulfment of apoptotic cells by phagocytes. Disruption of DIRCl by translocation $\mathrm{t}(2 ; 3)(\mathrm{q} 33 ; \mathrm{q} 21)$ is associated with familial clear cell renal cancer. The gene expression of GULP1 and DIRC1 in ESCC tumor tissues at 2q32.1-q32.2 was 0.89-fold and 1.27-fold, respectively, in our unpublished microarray dataset. Further study of candidate genes in this locus is necessary to verify their role in ESCC susceptibility. Another potential genetic 
susceptibility locus for ESCC at 10q23.33-q24.1 worthy of attention harbors one of the candidate genes located within the region, PLCE1 (phospholipase C, epsilon 1). Notably, $P L C E 1$ was recently reported independently to be associated with ESCC in the high-risk region of Northern China by a genome-wide association study (GWAS) (30-32). The same locus at 10q23 was identified independently by three different groups as risk factors for high ESCC incidence areas in China with a combined large sample size of 16,499 ESCC and 21,998 controls. It has been hypothesized that germline IBD regions represent low-penetrance factors for cancer predisposition (17). The potential cancer susceptibility loci identified in our data did not localize to loci of high penetrance cancer susceptibility genes such as BRCA1 at $17 \mathrm{q} 12, B R C A 2$ at $13 \mathrm{q} 14$, or TP53 at $17 \mathrm{p} 13-\mathrm{p} 15$, but reside at regions similar to PLCE1 at 10q23 with low-penetrance susceptibility for cancer development. Combining the data from shared IBD segments by pairing analysis and the microarray profiling allowed us to narrow down the candidate genes for validation. These studies support the biological relevance of at least four target genes residing within the IBD segments at 10q23.33-q24.1 and 16q11.2-q12.1 having significant differential expression in primary ESCC tumors. PLCE1 is a phospholipase responsible for hydrolysis of phosphatidyl-inositol 4,5-bisphosphate regulating 1,2-diacylglycerol downstream signaling and an effector of GTPases, such as, Ras (33). CYP2-C18, (cytochrome P450, family 2, subfamily $C$, polypeptide 18) located within a cluster of cytochrome P450 genes at 10q24, encodes one of the cytochrome P450 superfamily of enzymes, which are monooxygenases involved in drug metabolism and synthesis of cholesterol, steroids and other lipids. SIAH1 (siah E3 ubiquitin protein ligase 1) is an E3 ligase. It may play a functional role in Parkinson's disease development, regulation of the cellular response to hypoxia and apoptosis. GPT2 [glutamic pyruvate transaminase (alanine aminotransferase 2)] encodes an enzyme for amino acid metabolism and gluconeogenesis. Importantly, PLCE1, GPT2, SIAH1 and CYP2-C18, appear to play an important role in ESCC tumorigenesis. Further in-depth genomic studies on these genes and others residing in these critical IBD regions are needed to elucidate their roles in ESCC.

The increased length of germline genomic homozygosity associated with hereditary ESCC in Henan was observed. To strategically increase the power of the present study, we focused on high ESCC incidence region samples with ESCC $\mathrm{FH}^{+}$cases and with multiple ESCC cases within one family. The ancestries of Henan cases and control samples were also carefully matched to avoid false signals introduced by small differences in ancestry. However, the results were still limited by the small sample size. The importance of these IBD segments in the etiology and development of ESCC in highrisk areas requires further investigation with an expanded sample size for validation, and capture region targeted sequencing of the eight potential cancer susceptibility loci identified in this study is required to search and validate the importance of the disease-causing genetic variants responsible for familial ESCC development. Importantly, using a genetic IBD approach for the study of inherited ESCC, it is clear that host genetic susceptibility does indeed contribute to ESCC development in high-risk Henan.

\section{Acknowledgements}

We acknowledge the Research Grant Council of Hong Kong SAR, P.R. China for the funding to M.L.L.

\section{References}

1. Parkin DM, Bray F, Ferlay J and Pisani P: Global cancer statistics, 2002. CA Cancer J Clin 55: 74-108, 2005.

2. Tran GD, Sun XD, Abnet CC, et al: Prospective study of risk factors for esophageal and gastric cancers in the Linxian general population trial cohort in China. Int J Cancer 113: 456-463, 2005.

3. Zhang W, Bailey-Wilson JE, Li W, et al: Segregation analysis of esophageal cancer in a moderately high-incidence area of northern China. Am J Hum Genet 67: 110-119, 2000.

4. Qiao YL, Hou J, Yang L, et al: The trends and preventive strategies of esophageal cancer in high-risk areas of Taihang Mountains, China. Zhongguo Yi Xue Ke Xue Yuan Xue Bao 23: 10-14, 2001 (In Chinese).

5. Xibib S, Meilan H, Moller H, et al: Risk factors for oesophageal cancer in Linzhou, China: a case-control study. Asian Pac J Cancer Prev 4: 119-124, 2003.

6. Yokokawa Y, Ohta S, Hou J, et al: Ecological study on the risks of esophageal cancer in Ci-Xian, China: the importance of nutritional status and the use of well water. Int J Cancer 83: 620-624, 1999.

7. Yang CS: Research on esophageal cancer in China: a review. Cancer Res 40: 2633-2644, 1980.

8. Hu N, Dawsey SM, Wu M, et al: Familial aggregation of oesophageal cancer in Yangcheng County, Shanxi Province, China. Int J Epidemiol 21: 877-882, 1992.

9. Zhang G, Su M, Wang D, et al: Genetic heterogeneity of oesophageal cancer in high-incidence areas of Southern and Northern China. PLoS One 5: e9668, 2010.

10. Su H, Hu N, Shih J, et al: Gene expression analysis of esophageal squamous cell carcinoma reveals consistent molecular profiles related to a family history of upper gastrointestinal cancer. Cancer Res 63: 3872-3876, 2003.

11. Hu N, Roth MJ, Polymeropolous M, et al: Identification of novel regions of allelic loss from a genomewide scan of esophageal squamous-cell carcinoma in a high-risk Chinese population. Genes Chromosomes Cancer 27: 217-228, 2000.

12. Hu N, Wang $\mathrm{C}, \mathrm{Su} \mathrm{H}$, et al: High frequency of $C D K N 2 A$ alterations in esophageal squamous cell carcinoma from a high-risk Chinese population. Genes Chromosomes Cancer 39: 205-216, 2004.

13. Hu N, Wang C, Ng D, et al: Genomic characterization of esophageal squamous cell carcinoma from a high-risk population in China. Cancer Res 69: 5908-5917, 2009.

14. Hu N, Su H, Li WJ, et al: Allelotyping of esophageal squamouscell carcinoma on chromosome 13 defines deletions related to family history. Genes Chromosomes Cancer 44: 271-278, 2005.

15. Bacolod MD, Schemmann GS, Wang S, et al: The signatures of autozygosity among patients with colorectal cancer. Cancer Res 68: 2610-2621, 2008.

16. Broman KW and Weber JL: Long homozygous chromosomal segments in reference families from the centre d'Etude du polymorphisme humain. Am J Hum Genet 65: 1493-1500, 1999.

17. Assie G, LaFramboise T, Platzer P and Eng C: Frequency of germline genomic homozygosity associated with cancer cases. JAMA 299: 1437-1445, 2008.

18. Rudan I, Rudan D, Campbell H, et al: Inbreeding and risk of late onset complex disease. J Med Genet 40: 925-932, 2003.

19. Lebel RR and Gallagher WB: Wisconsin consanguinity studies. II: Familial adenocarcinomatosis. Am J Med Genet 33: 1-6, 1989.

20. Shami SA, Qaisar R and Bittles AH: Consanguinity and adult morbidity in Pakistan. Lancet 338: 954, 1991.

21. Kanai N, Fujii T, Saito K and Tokoyama T: Rapid and simple method for preparation of genomic DNA from easily obtainable clotted blood. J Clin Pathol 47: 1043-1044, 1994.

22. Purcell S, Neale B, Todd-Brown K, et al: PLINK: a tool set for whole-genome association and population-based linkage analyses. Am J Hum Genet 81: 559-575, 2007. 
23. Browning SR and Browning BL: High-resolution detection of identity by descent in unrelated individuals. Am J Hum Genet 86: 526-539, 2010.

24. Chan SH, Yee Ko JM, Chan KW, et al: The ECM protein LTBP-2 is a suppressor of esophageal squamous cell carcinoma tumor formation but higher tumor expression associates with poor patient outcome. Int J Cancer 129: 565-573, 2011.

25. Cheung AK, Ko JM, Lung HL, et al: Cysteine-rich intestinal protein $2(C R I P 2)$ acts as a repressor of NF- $\mathrm{KB}$-mediated proangiogenic cytokine transcription to suppress tumorigenesis and angiogenesis. Proc Natl Acad Sci USA 108: 8390-8395, 2011

26. Cheung AK, Lung HL, Hung SC, et al: Functional analysis of a cell cycle-associated, tumor-suppressive gene, protein tyrosine phosphatase receptor type $\mathrm{G}$, in nasopharyngeal carcinoma. Cancer Res 68: 8137-8145, 2008 .

27. Rudan I: Inbreeding and cancer incidence in human isolates. Hum Biol 71: 173-187, 1999.

28. Lander ES and Botstein D: Homozygosity mapping: a way to map human recessive traits with the DNA of inbred children. Science 236: 1567-1570, 1987.
29. Reversade B, Escande-Beillard N, Dimopoulou A, et al: Mutations in PYCRl cause cutis laxa with progeroid features. Nat Genet 41: 1016-1021, 2009.

30. Wang LD, Zhou FY, Li XM, et al: Genome-wide association study of esophageal squamous cell carcinoma in Chinese subjects identifies susceptibility loci at PLCE1 and C20orf54. Nat Genet 42: 759-763, 2010.

31. Abnet CC, Freedman ND, Hu N, et al: A shared susceptibility locus in PLCE1 at 10q23 for gastric adenocarcinoma and esophageal squamous cell carcinoma. Nat Genet 42: 764-767, 2010.

32. $\mathrm{Wu} \mathrm{C}, \mathrm{Hu} \mathrm{Z}, \mathrm{He} \mathrm{Z}$, et al: Genome-wide association study identifies three new susceptibility loci for esophageal squamous-cell carcinoma in Chinese populations. Nat Genet 43: 679-684, 2011.

33. Bunney TD and Katan M: Phospholipase C epsilon: linking second messengers and small GTPases. Trends Cell Biol Biol 16: 640-648, 2006. 